\title{
A Comprehensive Analysis On Efficient Multimedia Storage Mechanism In Public Cloud Environment With Secured Access
}

\author{
G. Megala ${ }^{a}$, S. Prabu ${ }^{\text {** }}$
}

${ }^{a}$ Research Scholar, School of Computer Science and Engineering, Vellore Institute of Technology, Vellore, Tamil Nadu, India. Email: megala.g39@gmail.com

b*Professor, School of Computer Science and Engineering, Vellore Institute of Technology, Vellore, Tamil Nadu, India. E-mail: sprabu@vit.ac.in

Article History: Received: 11 January 2021; Accepted: 27 February 2021; Published online: 5 April 2021

\begin{abstract}
Dynamic on-demand service has become the crucial part in cloud computing according to the user's requirements. Cloud storage is clearly the top choice for archiving media assets, by choosing the public cloud for active, deep archive needs. Video on demand, media distribution system in cloud allowing users to access video, facilitating immediate download and view of data without traditional video playback devices. On demand service of video requires infrastructure resources to handle large load fluctuations. Major challenges of video demand services are delays in simultaneous video uploading, processing, accessing and traffic caused by exhaust services. Also, there are concerns over the security of assets stored in a public cloud remains. In this paper, an ample analysis is presented on file storage encompassing fragmentation, compression, encryption security concerns in cloud storage and access. The limitations in the existing is also provided and hence outlined the research direction towards an efficient storage mechanism with a secured access providing a reliable media data transmission between data owner and users in public cloud environment.
\end{abstract}

Keywords: Video on Demand, Fragmentation, Compression, Encryption.

\section{Introduction}

Cloud computing is the on demand availability of computer system resources to the users without their direct management. It provides network access, resource pooling, elasticity, on demand self-service and measured service (pay per usage cost). All these services are provided by cloud service platform through the internet to the users. Cloud service providers contains storage servers, database servers providing infrastructure as a service by comprising virtualization. Cloud also offers platform as a service facility through web servers and software as a service via application interfaces. Through this web application interface the clients upload their data to store it in the remote servers of the cloud provider after successful authentication process. Advanced automation techniques are used to fulfill the requests of customers who procure cloud services. Agility is the key advantage of the abundance of cloud services.

Cloud implementation are generally classified into public cloud, private cloud and hybrid cloud. Customers gain access to hardware and software with the subscription based fee in the public cloud. Today IT businesses prefers a multi cloud approach, which uses more than one public cloud service. Though public clouds had proved themselves as less susceptible to attack, objections to the public cloud begins with cloud security. Integration of security policies and identity access management between customers and public cloud providers are the major concern. File storage and maintenance cost burden are controlled in the IT sectors and organizations with the help of cloud storage. Benefits of cloud storage are less storage cost, enlarged operational flexibility and optimized backup infrastructure.

\subsection{Motivation}

Video on demand, a broadband streaming service can be adopted using the increased internet traffic to distribute the video contents in a huge quantity over the network. This allows the users to stream the recorded video content provided by video media distribution system. Multimedia contents including video and audio file which are huge in volume are stored in a centralized cloud data center. Network caching can mitigate the flooded multimedia traffic growth called congestion and disruption of service. Public cloud storage offers media and entertainment organizations even greater benefits, such as additional cost savings, collaborative creative workflows that know no geographic limit and easy access to artificial intelligence and machine learning based tools in the cloud that can make stored media assets easier to search and thus more valuable. 


\subsection{Goal and Contributions}

The survey aims to graph the research on storage services provided by public cloud environment for multimedia file, an unstructured data having high dimensionalities. Future direction is to provide security for those media files storage and access in cloud platform. In order to achieve it, a survey has been done on various whitepapers related to storage, security and retrieval techniques. The main contributions of this paper are comprehensive investigation on storage methods in cloud, including fragmentation, segmentation and compression of video files, security challenges preserving privacy on data for efficient storage mechanism and accessing.

\section{Related Works}

Miguel Santiago-Duran, et. al, [1] presented a software pipelined systems for processing huge volume of data which are condensed into large virtual containers using parallel pattern and memory storage. A capsule of five gears such as, LZ4 for compression, AES to encrypt the data, SHA3 - 256 to calculate hash value verifying integrity of received content, IDA to split the data into multiple segments \& disperse and CURL which send content to cloud through Http stream; are encapsulated to the software pipelined collaborative system. Though this capsule container tool produce uniform velocities in processing data, reliability, resilience and load balancing are to be improved.

Zhao Y, et. al.,[2] surveyed data mining techniques to preserve privacy and proposed a Privacy Preserving Tensor based Multiple Clustering(PPTMC) technique in order to protect privacy of user while creating multiple clusters on cloud. PPTMC, an analytics and service based framework was proposed to collect data from cyberspace, physical space and social space to perform data object tensorizatrion in the data plane. Feature extraction and multiple clustering's are done in the analytic plane which is being serviced by service plane. A perturbation technique is also used for preserving privacy. This framework generated the encrypted clusters in the public cloud by calculating secure weighted tensor distance based on secure attribute weight ranking. Though the performance was less operative in high dimensional data

Anejionu, Obinna CD, et. al., [3] proposed a urban data system on spatial data, which is a medium scaled geospatial bigdata system for integrating and processing the data which are spatially activated, by monitoring and comparing the urban area performances to facilitate the intra city dynamics understanding. Components of the system include ETL feature manipulation engine, postgress SQL for processing and analyzing spatial data, web visualization, and cloud based data warehouses such as snowflake, EU to store the content protectively with recoverable facility with the combination of Kerberos authentication providing security. The Strava dataset, a small area dataset of cycling, running and walking activities are uploaded to this automated system for processing, analyzing and visualization outputs through API.

Amazon web services [4] provides many different services such as compute, storage, database, developer tools, networking, security, identity \& compliance, media services, application integration, etc., so as to provide the customer as pay per usage concepts. Amazon s3 is the serverless storage service predominantly used for object file storage in a bucket. Whereas amazon EC2 is a server based computation service providing secure, scalable compute capacity in cloud. Optimized storage service for media files are done by AWS elemental media store. AWS snowball service is to provide transportation of data in petabyte scale using secure application. Amazon cloud permits the customers to build a flexible cloud directory and cloud-native directories to store and organize the data hierarchically in multiple dimensions. Though amazon provide plenty of services, they sets default limits on resources varying from region to region.

Nasuni [5] presents a Global File System (GFS) for storing unstructured data as a object storage which provides a collaborate file across multiple sites, NAS and remote office file servers. Object storage uses the approach of Write Once Read Many (WORM) so as to prevent erasure. GFS unlocks the potential of object storage compared to file storage use cases such as archiving, data center and multi-site file collaboration. Benefits of object storage are low egress cost, high performance file access, familiar hierarchical folder structure, application compatibility. Though it limits to offer native data protection.

Goli-Malekabadi Z; et. al., [6] deployed a model based on NoSQL databases, the Document-based databases Cloud storage for storing the healthcare data to afford high scalability, availability and quick retrieval of complex unstructured data. Document based database performs write operation in write once and read many approach of different sizes. This database SQL server uses indexing techniques for fast query processing and retrieval. Document based database includes shard feature which increases the data recovery operation speed but slows down the write operation. 
Becker, et. al.,[7] performed cloud based object storage of images so as to provide elastic storage at low cost using techniques such as Meta Raster Format (MRF) and Limited Error Raster Compression (LERC), a lossy compression algorithm utilizing few cpu cycle for compression and decompression. Geospatial images are stored in MRF format, a high performance web tile service storage format which splits the rater dataset into three files such as metadata file, data file and index file. Those compressed images are transmitted as blocks of pixel to client application including the checksum providing integrity. The delay between the client request and cloud service provider response tried to reduce but had a greater latency for data access.

Mehdi Sookhak, et. al.,[8] implemented a data storage scheme, Remote Data Checking (RDC) with divide and conquer tables data structure in order to authenticate the data integrity of outsourced information with algebraic signature properties. This scheme was developed to allow the users to update the block dynamically on the verifier and server auditing large scale files. This method has setup phase, challenge and response, and verification detecting the misbehavior of cloud service provider such as replay attack by integrity verification of the outsourced data. This single server auditing method detects the data corruption overcoming the data recovery capability.

Hitachi Vantara [9] illustrated how technologies leveraged to drive business, managing large volume of data and insight of business drive with Hitachi vantara pentaho platform. Raw data has been staged in Apache Hadoop distribution, a framework for high volume unstructured data processing across distributed file system. Hitachi has made their data available in amazon redshift, which performs Extract transform and load (ETL) operation on big data, including map reduce technique via Hive data warehouse layer and orchestrated data using pentaho data integration.

Renny shen [10] illustrates the solution provided by f5 to build a flexible cloud storage model for cloud, using existing capacity rather than purchasing it. Object based storage platform with a global namespace is used to integrate storage capacity and also provides a storage gateway to enable the movement of files to the cloud, an automated storage tiering. To establish flexible cloud storage model, file virtualization solution is used so-called ARX cloud extender, a software which is installed in the windows based web server. This provides file access on private or public cloud and translate file access to suitable web service application protocol interface.

Zheng Xu, et. al.,[11] implemented front + back pattern to address the redundant structure of public security information system. This method provides a storage environment to perform mining operation and semantic analysis. Videos captured in video surveillance system are divided into parts by considering time as the key factor; transferred to data center at back for complex computation in a unified standard format where it is being stored in a distributed file systems. Map reduce, spark, storm, virtualization are involved in distributed processing model performing content analysis, semantic modelling \& reasoning and complex data analytics.

Xuan Chen[12] adopted the concept of fragmentation combined with selective encryption, a light weight encryption approach based on AES to selectively protect the social photo sharing in the current social big data knowledge system. A social media image is mapped into different spaces and transformed image is fragmented into two parts; where one is randomly selected, encrypted and stored in one platform as a protected small fragment. Other fragment i.e., public large fragment is stored in other social platform. At the destination side, protected small fragment and public large fragment from different platforms are decrypted and mapped, defragmented to form the social image which does not support for multiple party communication.

Jegadeeswari S et. al., [13] proposed dynamic bloom filtering hashing technique, a cloud based data storage technique to protect the outsourced chunked data from the attackers or unauthorized ones. Data fragmentation model performs horizontal and vertical fragmentation based on key and non-key attributes so as to identify the confidentiality of attributes such as high, medium or low. Bloom filter data structure stores the sensitive fragment securely using hash function in a dynamic hashing scheme and performs mapping for retrieval access by user. The author has focused more on fragmentation, storing and accessing. But the author has not concentrated in providing security (i.e. encryption) of those fragmented data which may lead to unauthorized access.

Tseng-Yi Chen, et. al.,[14] implemented multigrained block management strategy over a Phase change memory (PCM) based storage system to resolve the issues raised during fragmentation of file system. A sub block management is performed with the stack structure to manage multiple size blocks of each file and thus enhanced space utilization of inode-based file system. Internal fragmentation is performed to form sub blocks of fixed size which are stacked by dynamic inode allocation, maintaining dynamic allocation status, compaction and space reclamation of inode in the inode translation table so as to resolve external fragmentation but has not addressed the continuous space allocation. 
Muhammad Haroon et. al.,[15] proposed Bag of visual words (BoVW) framework to detect the boundary between the shots and scene. Vector of Linearly Aggregated Descriptors (VLAD) is used to slice the videos into shots for further indexing and searching. Entropy differences are computed to detect shot boundary, where the key frames are selected based on the larger entropy. Detected key points are represented as key descriptors which is a time consuming factor. Two alike key point descriptors which are distorting are assigned with different visual words and feature quantization are performed. Sum of the difference between outstanding descriptors are computed with visual words and concatenated into single vector in VLAD model; but there was less accuracy obtained in shot boundary detection.

Liang Zhao, et. al., [16] performed segmentation on moving objects by computing moving vector interpolation for intra coded prediction unit, preprocessing the vectors, clustering the blocks of non-zero moving vectors using connectivity component labelling. Person-vehicle classification is also implemented in two phases. Bag of spatio temporal HEVC syntax words are trained to the model in training phase. In testing phase, spatio-temporal information are extracted to get feature descriptors. Though the proposed model suffers to distinguish moving persons and vehicles in a frame.

Jiangyun $\mathrm{Li}$, et. al., [17] proposed a semantic video segmentation model so as to strengthen inter frame and intra frame features for segmentation prediction. The interframe features of same objects are enhanced across adjacent frames to predict the temporal consistency using adjacent attention module. Feature correlations are modeled in spatial attention module which aggregates features of interframe within single instance to predict the spatial consistency. Upsampling operations were complex to optimize the boundary.

Yang Yu, et. al.,[18] addressed the pedestrian video segmentation method by constructing spatio temporal graph cut segmentation framework. Temporal correspondence of boundary box sequence of pedestrian are provided by optimal transportation minimization framework. Edge sticky superpixel (ESS) maintains the spatial consistency of object boundary and reduce redundancies to preserve boundaries. Though ESS are equality sized, it lacks the ability to handle occlusions.

Abir Jaafar Hussain, et. al.,[19] surveyed on eradicating temporal redundancies in video sequences and fast block matching algorithm for video compression, a motion compensation predictive coding. Author studied dividing video into a set of non-overlapped macro blocks which is then compared with the search area to find the best match macroblock. Full search is done with same resolution of predicted frames in lossless block matching. Whereas less quality is achieved with reduced time complexity in lossy block matching algorithm compression technique.

Ratnajit Mukherjee, et. al.,[20] encoded dynamic scene range by perceptual transfer function using uniform color opponent space. In encoding, range of input pixel values are mapped to a range of output code values. Logarithmic transfer function adapted to encode whole range of visible luminance into n-bit code value. Threshold vs intensity function is derived based on the boundaries for intensity channel encoding but the quantization errors are not reduced.

Mariana Afonso, et. al.,[21] proposed a framework on spatio-temporal resolution adapted video compression involving integration of spatial and temporal adaption, computing features from unencoded input video frames in Quantization resolution Module determining optimal resolutions to encode the frames. For resolution upscaling and frame repetition at decoder, CNN based on super resolution model is constructed which is having high complexity for spatio-temporal resampling.

Zhibo Chen, et. al.,[22] modeled Pixel Motion CNN (PMCNN) to perform spatio temporal coherence by predictive coding, iterative analysis and binarization which are jointly optimized in the training phase. Exploiting invariance between adjacent frames, allocating different bits to different blocks are involved in temporally progressive coding in testing phase. Performance is less achieved as the entropy coding was not implemented.

Voundi Koe Arthur sandor, et. al., [23] decentralized the multi authority attribute, solving key escrow problem using decisional bilinear diffi-hellman assumption in mobile cloud data storage by removing the central authority without using and global identity of user. Speed of encryption and decryption, minimum computation overhead were achieved among user and multiple attribute authorities. It has been implemented based on complete trust on data owner which might lead to security concerns.

Hassan Elkamchouchi, et. al.,[24] used VCMAB, substitution method for encrypting and VCMTH, permutation method to perform shuffling of different block sizes, involving RGB colour channel swapping based 
on key, Mpeg-2 compression, Scrambling of blocks based on preselected frame, XORing with the previous encrypted frames; thus generated keystreams for video frame encryption by achieving confusion and diffusion. These chaotic algorithms are more sensitive to cropping attacks as indicated by low PSNR value and slight variations in decrypting key may result a corrupted video.

Yao-Tang Changa, et. al.,[25] proposed Chaos/Binary mapping Algorithm (CBmA) to protect the video content during transmission on the basis of PSNR degradation by implementing parsing the codestream, shuffling and scrambling the optical wavelength which is controlled by Arrayed Waveguide grating (AWG) and optical switch matrix to dynamically change optical wavelength of authorized users. This lead to a substantial drop in PSNR value in transmission which made unidentifiable by unauthorized users.

YiqiTew, et. al.,[26] implemented a two-level authentication scheme which combine encryption and authentication on a compressed video with syntax element set representing the authentication code generation information on each sliced video embedded to it so as to detect the tampered one. This joint scheme is able to distort the video quality which can be restored back to original HEVC format achieving confidentiality by performing perceptual masking and thus verifying the integrity of video. Introduced distortion makes outline noticeable when sign bit in luminance channel is randomized which might lead to compromised by adversary to manipulate the slice.

Shouxin Zong, et. al.,[27] proposed an analog transmission scheme, softcast to provide video quality degradation by unequal allocation of power among chunk. Original signal reconstructed without metadata at the receiver end based on the chunk based blind elimination method which tried to eliminate cliff effect without using power scaling factor, though there was occurrence of distortion. Although small chunks achieved less performance resulting in less noise suppression due to bandwidth and power loss.

Jonathan Kua, et. al., [28] deployed adaptive chunklets(technique dividing DASH video chunk HTTP request range) instead of fixed chunklet so as to reduce the bandwidth with the combination of Flow Queue-Controlled Delay (FQ-CoDel) which maximizes the quality of experience in the presence of cross traffic in the DASH systems. This method mitigates delay spikes, building queue, loss of packets in latency sensitive flow with flow isolation, prioritizing traffic for VoIP having low bitrate flow.

Kun He, et. al.,[29] implemented an autonomous update Key Policy ABE (KP-ABE) to allow users to have dynamic membership update or revoke or regenerate their keys separately without making all group members to be involved in it so as to provide flexible authentication and effectual authorization for VoD services. Computation cost is high as the private keys cannot be regenerated for registered users without accomplishing revoking and updating.

Minseok choi, et. al., [30] proposed a technique to make decision on the receiving chunk size dynamically using lyapunov optimization. Caching node decisions are done using markov decision process at the receiver end to obtain the desired file. This decision processes are made to provide high video quality by maximizing streaming quality with reduced delay. Dynamic video delivery has been addressed for wireless caching networks in two different timescales considering mobility of user but limits to playback latency which is the queuing delay.

White paper on Alibaba cloud [31] implemented a cloud based video surveillance solution in different modules with high complexity and account access key verification for accessing directly the OSS. Server load balancer in the Video storage module is used to upload the video streams with an uplink bandwidth in GB range to ECS instances where the segmented videos are stored as object in a distributed manner. Video viewing module submits the received request from user to the server, performs device verification, searches the index of video and retrieves it. Management services such as user information management, device management and user verification are performed by system management module.

Angelo furfaro et. al., [32] has simulated the bandwidth flooding attacks handled by the hybrid filter mechanism developed involving differentiated service policies integrated with stopIt technique exploiting network filters on routers. An alerts has been sent by the router to the server when the traffic flow exceeds bandwidth and looks for malicious sources by log monitoring but it is not realistic to large scale topology having more response time thus decrease in performance occurred. 


\section{Problem Formulation}

Considering storage design aspects studied in related works, the problem statement has been formulated using the model aiming to address the issues faced by media files while storing in public cloud environment and security issues. This model tries to overcome the network resource, bandwidth exhaustion while storing and retrieving the video content to/from the cloud.

\section{System Model}

Objective of the proposed system is to develop an efficient mechanism for storing video files in the public cloud environment. It is to be served for the cloud users on demand basis with reduced latency, less time complexity, high security and accuracy. Data owner initializes the service level agreement with the cloud service provider. Service provider sends the final SLA generated to data owner based on the clients' requirements and provides the service accessibility. Through the web application interface, the Data owner uploads the huge volume of video files to the cloud server. Here the media contents are being fragmented based size and produces fragments (also called chunks). Those fragments are then compressed and encrypted to secure the media. Those encrypted files are outsourced with key to store it in the remote servers cloud service provider as shown in Fig. 1.

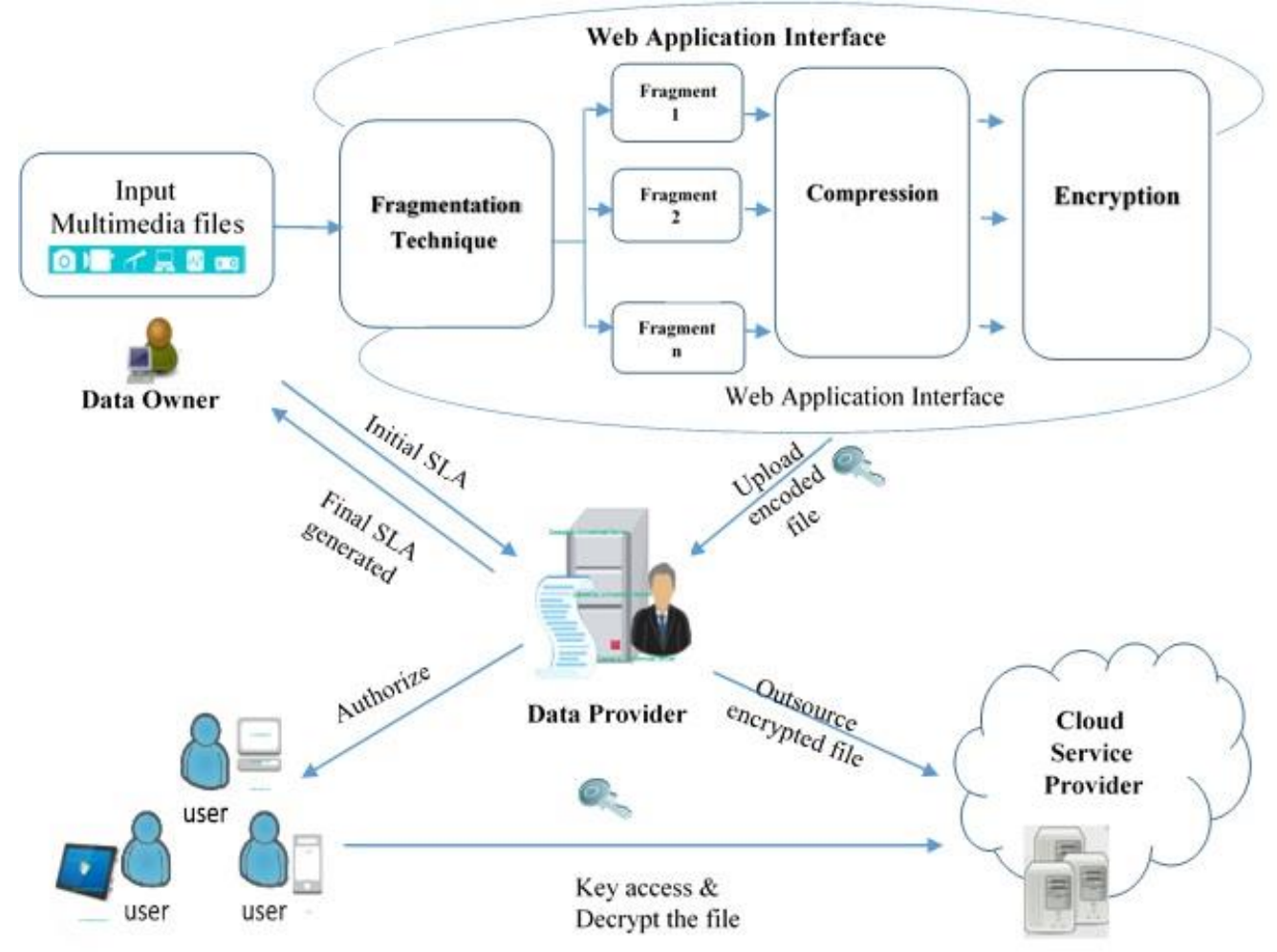

Figure 1. Workflow Diagram of System Model

Fragmentation is performed on the inputted video data to produce the chunks. This can be done in two ways, horizontal fragmentation and vertical fragmentation considering the size and time length as key parameter. These chunks are then encoded using the compression techniques involved with the combination of motion estimation of the frames. There are several compression techniques available, such as Particle swarm optimization, block matching using Discrete cosine transform (DCT) and Discrete wavelet transform (DCT), Principal component analysis, CNN based super resolution, vector quantization, entropy encoding and so on. Considering the time complexity an efficient encoding method is performed on those fragmented chunks to produce encoded stream. There are several encryption techniques such as full layered encryption, scrambling based encryption, selective encryption, perceptual encryption, chaotic based encryption, shifting and region permutation. After identifying the best encryption method, those highly lossless compressed chunks are then encrypted with less computation time and high efficiency. These encrypted chunks are then outsourced to the cloud service provider for on demand access.

Video data service provider shares the public key to the data owner for the storage; whereas data user sends a request for video content access to the service provider. Video on demand data provider validates, authorizes and 
provides an authenticated key to the requested users so providing video content delivery on demand basis with high security, accuracy and fast access.

\section{Conclusion}

This paper presents a comprehensive study on secure and efficient data storage mechanism in public cloud environment. A video content management system is designed to upload a massive volume of video data to the cloud environment by fragmenting, compressing, converting the video files into appropriate bit rate, frame rate and resolution in order to make video file available for quality access with the combination of efficient encryption and security algorithms considering time complexity, computation complexity and latency.

\section{Declaration of Competing Interest}

The authors declare that they have no known competing financial interests or personal relationships which have or could be perceived to have influenced the work reported in this article.

\section{References}

1. Santiago-Duran, M., Gonzalez-Compean, J.L., Brinkmann, A., Reyes-Anastacio, H.G., Carretero, J., Montella, R., \& Pulido, G.T. (2020). A gearbox model for processing large volumes of data by using pipeline systems encapsulated into virtual containers. Future Generation Computer Systems, 106, 304319.

2. Zhao, Y., Tarus, S.K., Yang, L.T., Sun, J., Ge, Y., \& Wang, J. (2020). Privacy-preserving clustering for big data in cyber-physical-social systems: Survey and perspectives. Information Sciences, 515, 132-155.

3. Anejionu, O.C., Thakuriah, P.V., McHugh, A., Sun, Y., McArthur, D., Mason, P., \& Walpole, R. (2019). Spatial urban data system: A cloud-enabled big data infrastructure for social and economic urban analytics. Future Generation Computer Systems, 98, 456-473.

4. Whitepaper on Overview of Amazon web services, (2020).

5. https://docs.aws.amazon.com/whitepapers/latest/aws-overview/aws-overview.pdf\#introduction

6. Whitepaper on Nasuni-e-guide-Object storage vs File storage, (2018).

7. https://info.nasuni.com/hubfs/Nasuni.com-assets/e-Guides/Nasuni-e-Guide-Object-Storage-vs-FileStorage-6-Reasons-Best-of-Both.pdf?hsLang=en

8. Goli-Malekabadi, Z., Sargolzaei-Javan, M., \& Akbari, M K. (2016). An effective model for store and retrieve big health data in cloud computing. Computer methods and programs in biomedicine, 132, 7582.

9. Becker, P., Plesea, L., \& Maurer, T. (2016). Optimizing cloud based image storage, dissemination and processing through use of MRF and LERC. International Archives of the Photogrammetry, Remote Sensing \& Spatial Information Sciences, 15, 41.

10. Sookhak, M., Yu, F.R., \& Zomaya, A.Y. (2017). Auditing big data storage in cloud computing using divide and conquer tables. IEEE Transactions on Parallel and Distributed Systems, 29(5), 999-1012.

11. Whitepaper on Big Data at Cloud Scale by Hitachi, (2019). https://www.hitachivantara.com/enus/pdfd/white-paper/big-data-at-cloud-scale-whitepaper.pdf

12. Whitepaper on Building a cloud-enabled File Storage Infrastructure. https://www.f5.com/pdf/whitepapers/cloud-enabled-storage-wp.pdf

13. Xu, Z., Mei, L., Hu, C., \& Liu, Y. (2016). The big data analytics and applications of the surveillance system using video structured description technology. Cluster Computing, 19(3), 1283-1292.

14. Chen, X. (2020). Security-preserving social data sharing methods in modern social big knowledge systems. Information Sciences, 515, 404-416.

15. Jegadeeswari, S., Dinadayalan, P., \& Gnanambigai, D. (2019). Efficient Dynamic Bloom Filter Hashing Fragmentation for Cloud Data Storage. Cybernetics and Information Technologies, 19(1), 53-72.

16. Chen, T.Y., Chang, Y.H., Yang, M.C., Chen, Y.J., Wei, H.W., \& Shih, W.K. (2015). Multi-grained block management to enhance the space utilization of file systems on PCM storages. IEEE Transactions on Computers, 65(6), 1831-1845.

17. Haroon, M., Baber, J., Ullah, I., Daudpota, S.M., Bakhtyar, M., \& Devi, V. (2018). Video scene detection using compact bag of visual word models. Advances in Multimedia, 2018.

18. Zhao, L., He, Z., Cao, W., \& Zhao, D. (2016). Real-time moving object segmentation and classification from HEVC compressed surveillance video. IEEE Transactions on Circuits and Systems for Video Technology, 28(6), 1346-1357.

19. Li, J., Zhao, Y., Fu, J., Wu, J., \& Liu, J. (2019). Attention-guided network for semantic video segmentation. IEEE Access, 24(7), 140680-140689. 
20. Yu, Y., Makihara, Y., \& Yagi, Y. (2019). Pedestrian segmentation based on a spatio-temporally consistent graph-cut with optimal transport. IPSJ Transactions on Computer Vision and Applications, 11(1), 1-17.

21. Hussain, A.J., \& Ahmed, Z. (2019). A survey on video compression fast block matching algorithms. Neurocomputing, 335, 215-237.

22. Mukherjee, R., Debattista, K., Rogers, T.B., Bessa, M., \& Chalmers, A. (2018). Uniform color spacebased high dynamic range video compression. IEEE Transactions on Circuits and Systems for Video Technology, 29(7), 2055-2066.

23. Afonso, M., Zhang, F., \& Bull, D.R. (2018). Video compression based on spatio-temporal resolution adaptation. IEEE Transactions on Circuits and Systems for Video Technology, 29(1), 275-280.

24. Chen, Z., He, T., Jin, X., \& Wu F. (2019). Learning for video compression. IEEE Transactions on Circuits and Systems for Video Technology 30(2), 566-76.

25. Sandor, V.K.A., Lin, Y., Li, X., Lin, F., \& Zhang, S. (2019). Efficient decentralized multi-authority attribute based encryption for mobile cloud data storage. Journal of Network and Computer Applications, 129, 25-36.

26. Elkamchouchi, H., Salama, W.M., \& Abouelseoud, Y. (2019). New video encryption schemes based on chaotic maps. IET Image Processing, 14(2), 397-406.

27. Chang, Y.T., Lin, Y.C., \& Wang, W.H. (2019). Intelligent shuffling cryptography with dynamic AWG/switch matrix for video transmission in WDM-PON network. Journal of Intelligent \& Fuzzy Systems, 36(2), 1009-1022.

28. Datta, D., Mishra, S., \& Rajest, S.S. (2020). Quantification of tolerance limits of engineering system using uncertainty modeling for sustainable energy. International Journal of Intelligent Networks, 1, 1-8. https://doi.org/10.1016/j.ijin.2020.05.006

29. Zong, S., Gao, S., Tu, G., Zhang, C., \& Chen, D. (2019). A metadata-free pure soft broadcast scheme for image and video transmission. Signal Processing: Image Communication, 78, 359-367.

30. Kua, J., Armitage, G., Branch, P., \& But, J. (2019). Adaptive Chunklets and AQM for HigherPerformance Content Streaming. ACM Transactions on Multimedia Computing, Communications, and Applications (TOMM), 15(4), 1-24.

31. He, K., Chen, J., Zhang, Y., Du, R., Xiang, Y., Hassan, M.M., \& Alelaiwi, A. (2017). Secure independentupdate concise-expression access control for video on demand in cloud. Information Sciences, 387, 7589.

32. Choi, M., No, A., Ji, M., \& Kim, J. (2019). Markov decision policies for dynamic video delivery in wireless caching networks. IEEE Transactions on Wireless Communications, 18(12), 5705-5718.

33. Whitepaper on Empowering multimedia streaming - The cloud approach, Alibaba cloud. http://alicloudcommon.oss-ap-southeast-1.aliyuncs.com/Whitepaper\%20\%20Empowering\%20Multimedia\%20Streaming.pdf

34. Furfaro, A., Pace, P., \& Parise, A. (2020). Facing DDoS bandwidth flooding attacks. Simulation Modelling Practice and Theory, 98, 101984. 